\title{
Evolución y desafíos en la formación médica de posgrado en Brasil
}

\author{
Evolution and challenges in postgraduate medical training in Brazil
}

\author{
Antônio Fernandes Lages ${ }^{1}$, Tania Maria Marcial²
}

\section{Resumen}

La especialización médica es un gran desafío porque necesita preparar un profesional recién egresado en el especialista que la población quiere y necesita para asegurar mejores condiciones de atención a la salud. La construcción de un sistema para la capacitación de estos profesionales es un gran desafío dada la característica de la formación en servicio, es decir, los profesionales en formación trabajan directamente en la asistencia, dependiendo de instalaciones adecuadas, aparatos, tutores médicos y un programa teórico que garantice el desarrollo del aprendizaje según los parámetros técnicos más actuales. Un desafío adicional es la cantidad y diversidad de instituciones involucradas, desde hospitales universitarios con tradición docente hasta hospitales privados donde el desempeño se limita a la prestación de servicios. En este artículo, buscamos mostrar la trayectoria de la construcción de este sistema en Brasil, que desde hace cerca de 40 años se ha mejorado y ampliado ante la creciente demanda de atención de calidad por parte de la población.

Palabras clave: residencia médica; residencia médica en Brasil; formación médica de posgrado; especialización médica.

\begin{abstract}
Medical specialization is a challenge because it needs to prepare a newly graduated professional as the specialist that the population wants to ensure better conditions in health care. The construction of a system for training these professionals is a challenging given the characteristic of in-service training where professionals work directly with patients, depending on adequate facilities, equipment, medical tutors, and theoretical programs that guarantee the development of learning within the most current technical parameters. An additional challenge is the number and diversity of institutions involved, including university hospitals that have a tradition of teaching and private hospitals where the performance is based on practice. We seek to show in this article the trajectory of the construction of this system in Brazil, which for about 40 years has been improving and expanding to fulfill the population's growing demand for quality care.
\end{abstract}

Keywords: Medical residence; medical residency in Brazil; postgraduate medical training; medical specialization.

Fecha de envío: 2021-10-26 - Fecha de aceptación: 2021-12-21

Brasil es un país grande, con 213 millones de habitantes (IBGE -2021), cerca de $48 \%$ de la población de América del Sur (Medidores de países, 2021), de distribución heterogénea y con una gran variación en el índice de desarrollo entre las diferentes regiones (IBGE,2021). Este hecho implica un gran desafío respecto a la cobertura de calidad en el servicio de salud para toda la población. El sistema de salud en Brasil experimentó un cambio radical en 1988, con la promulgación de la nueva constitución federal que creó el Sistema Único de Salud -SUS. (Boletín Oficial de La Unión, 1988), lo cual garantiza a la población el acceso universal y gratuito a la asistencia sanitaria - que abarca desde servicios en atención básica hasta cuidados terciarios de alta complejidad - costeada por el estado. La prestación de servicios es realizada por servicios públicos, filantrópicos y también por sectores privados contratados para complementar la asistencia, también contamos con la llamada salud complementaria, que consistente de entidades que ofrecen planes de salud privados, que actualmente llegan al 21,2\% de la población (48 millones de usuarios) (SIB/ANS/MS, 2021).

Brasil tiene 371 facultades de medicina que gradúan cerca de 35558 médicos anualmente (Ministerio de Educación de Brasil, 2021). El Consejo Federal de Medicina de Brasil (CFM) reconoce

(1) Facultad de Ciências Médicas de Minas Gerais - Maternidad UNIMED BH. Asociación de Apoyo a la Residencia Médica de Minas Gerais - AREMG. Belo Horizonte - MG - Brasil (2) Facultad de Medicina y Ecología Humana - FASEH. Vespasiano-MG - Brasil Autor de correspondencia: aflages@hotmail.com 
55 especialidades médicas con periodos de formación de 2 a 6 años según la especialidad. Con la gran evolución de la medicina, demandando una formación cada vez más especializada, estos egresados buscarán mayoritariamente una residencia médica para especializarse antes de iniciar su vida profesional.

En Brasil, la residencia médica se creó en la década de 1940, en el Hospital de Clínicas de la Universidad de São Paulo (figura 1) y en el Hospital de Servidores del Estado de Río de Janeiro, inspirada en la filosofía del modelo norteamericano, una vez que hasta ese año la mayoría de los médicos brasileños obtuvieron su formación como especialistas en ese país (Sousa, 1988).

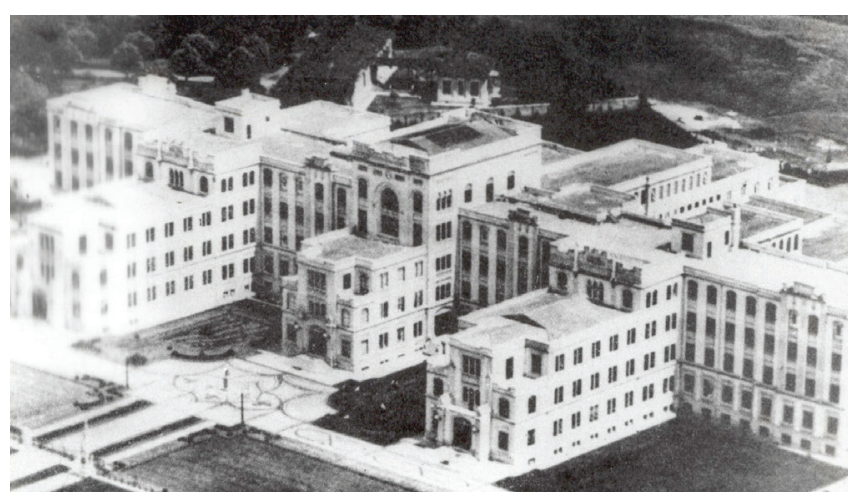

Figura 1: Hospital de Clínicas de la Universidad de São Paulo - USP primer hospital en Brasil con un programa de residencia médica en 1945.

Fuente: https://imagens.usp.br/wp-content/uploads/BIM0193.jpg

La creciente apertura de nuevas facultades de medicina en los años 1970 tuvo como consecuencia una rápida expansión de esos programas, no siempre con características de una enseñanza de posgrado, sino que en muchos casos, como una manera de obtener mano de obra a bajo costo. Este hecho ha motivado un gran movimiento hecho por médicos residentes a favor de la reglamentación de la residencia médica. En 1977 fue creada la Comisión Nacional de Residencia Médica (CNRM), una entidad independiente con representación de segmentos de la sociedad, responsable de reglamentar a la residencia médica en Brasil (Ministerio de Educación de Brasil, 1977).

La residencia médica es una modalidad de formación de posgrado dirigida a médicos, bajo la modalidad de curso de especialización. La residencia funciona en instituciones de salud, bajo la guía de profesionales médicos con alta calificación ética y profesional, siendo considerada el "estándar de oro" de la especialización médica en Brasil. Desde julio de 1981, con la Ley N6932, el término"residencia médica" sólo debe utilizarse para los programas acreditados por el CNRM (Ministerio de Educación de Brasil, 1981).

Con el crecimiento en el número de programas y por las dificultades para monitorearlos en los distintos estados del país, en 1987 se crearon los Comités Estatales de Residencia Médica (CEREM) (Ministerio de Educación de Brasil, 1987). Los CEREM son considerados representantes del CNRM en los Estados, y cada institución que administra programas de residencia medica cuenta con una Comisión de Residencia Medica (COREME) que actúa a su vez como representación del CNRM en la institución. Los CEREM actúan como intermediario entre los Comités de Residencia Médica de las instituciones - COREME y el CNRM. Los CEREM son muy activos en la organización y realización de inspecciones periódicas de los programas, reciben e investigan denuncias de irregularidades y participan en las reuniones plenarias mensuales del CNRM, actuando en las discusiones de todos los problemas y proyectos que involucran a la residencia medica. En 2012 se creó el banco nacional de evaluadores, (Ministerio de Educación de Brasil, 2012). y los programas pasaron a ser inspeccionados y evaluados por médicos inspectores aprobados en un proceso de selección.

Los COREME son encargadas de ofrecer, como parte de su servicio, las condiciones adecuadas para la buena formación del médico residente, siendo responsable de la aprobación del cuerpo de preceptores, de hacer cumplir las normas del CNRM y, en última instancia, de certificar al final de la residencia médica que el médico es entonces un especialista calificado para actuar en el mercado laboral. El reglamento de residencia médica establece los derechos y deberes de los médicos residentes, entre ellos, cumplir con una carga de trabajo semanal de 60 horas, incluyendo un máximo de 24 horas semanales de guardia, tener 24 horas de descanso semanal y 30 días consecutivos de vacaciones(Ministerio de Educación de Brasil, 1981); baja por maternidad de 120 días, baja por paternidad de 5 días, descanso mínimo de 6 horas tras la guardia nocturna(Ministerio de Educación de Brasil, 2011), y prohibición de realizar la guardia en la distancia (Ministerio de Educación de Brasil, 2010). Otras situaciones no previstas por la CNRM deben estar incluidas en el Reglamento de la COREME, tales como: tiempo máximo que un médico residente puede estar en licencia, y pasado este período, independientemente de la justificación, será excluido del programa. La mayoría de las instituciones colocan este período de un año. También el período de licencia por luto, matrimonio $\mathrm{u}$ otras situaciones no previstas en las leyes y resoluciones de la CNRM, quedan a criterio de la institución.

Las asociaciones de médicos residentes jugaron un papel fundamental en el desarrollo de los programas de residencia médica en Brasil. La primera instancia se encuentra dentro de las instituciones con las asociaciones locales de médicos residentes, teniendo sus representaciones dentro de las asociaciones estatales y en la Asociación Nacional de Médicos Residentes, la ANMR. Este fue fundado en 1967, durante la dictadura militar (ANMR, 2013). En los primeros años de su existencia, la entidad sirvió como foco para el 
resurgimiento del movimiento médico nacional. Desempeñó un papel fundamental en la regulación de la residencia médica, como modalidad de posgrado, y tiene plena participación en el CNRM.

El estado de Minas Gerais, ubicado en la región sureste de Brasil (Figura 2) es el segundo estado más grande de Brasil en términos de población, que tiene más de 20 millones de habitantes, teniendo la tercera economía más grande de Brasil (IBGE, 2021). La Asociación de Apoyo a la Residencia Médica de Minas Gerais - AREMG fue fundada en noviembre de 2002 por médicos representantes de la mayoría de las instituciones vinculadas a la residencia médica ubicadas en este estado. Su propósito es incentivar a la residencia médica en el estado de Minas Gerais, contribuyendo a mejorar la calidad de los programas de residencia y formación médica. AREMG apoya las actividades de las instituciones miembros que ofrecen programas de residencia médica. Entre las actividades desarrolladas se destacan: realización de un proceso de selección unificado para la residencia médica (AREMG, 2021), promoción de cursos de perfeccionamiento para residentes y tutores, asistencia y apoyo para la acreditación de nuevos programas y mantenimiento de los existentes.

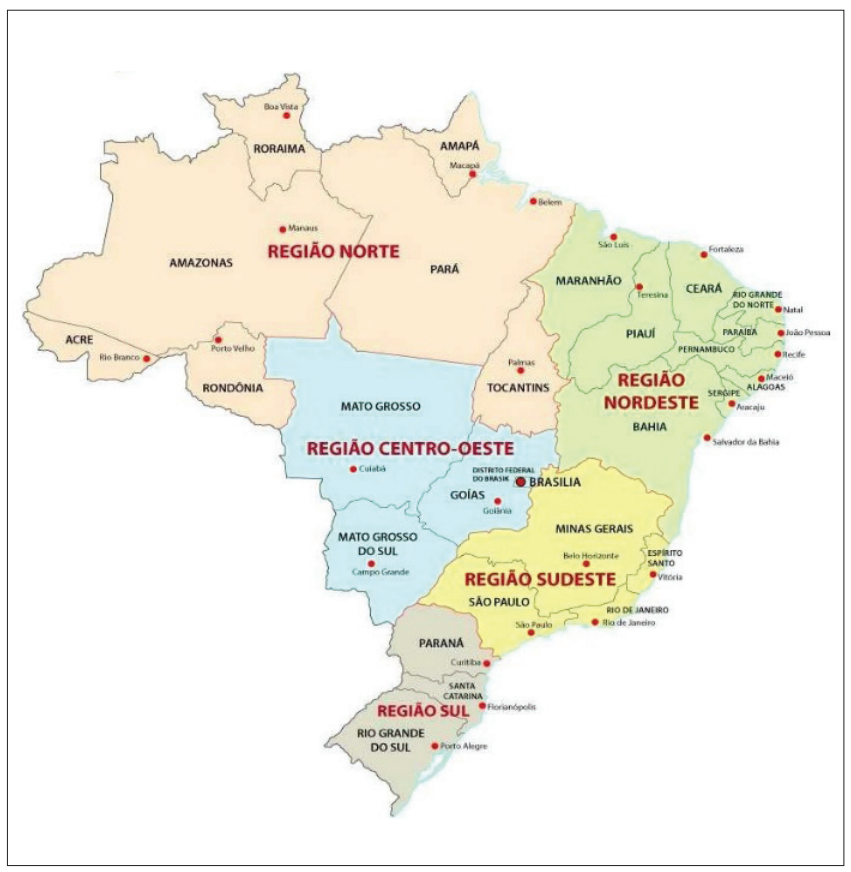

Figura 2: En las regiones sudeste y sur, se concentra el 59,2\% de los programas de residencia médica en Brasil. AREMG está ubicada en el estado de Minas Gerais, que pertenece a la región sudeste.

En Brasil se considera la residencia médica uno de los determinantes que condicionan la distribución geográfica de los médicos, no siempre coincidiendo con la distribución considerada socialmente adecuada. Al analizar la distribución geográfica de los espacios ocupados, encontramos una distorsión, ya que el 59,2\% de estos espacios se ubican en los estados de las regiones sur y sudeste del país, que son los más industrializados y con mayor tasa de desarroIlo, mientras que solo El 40,8\% se ubica en los estados del norte, noreste y centro oeste, las regiones más desfavorecidas (USP, 2018).

Existen evidencias de que la concentración de programas de residencia en las regiones sur y sudeste son factores que impulsan la distribución geográfica desigual de los médicos en el país. Se sugiere que las políticas de captación y retención de médicos basadas en la implementación de programas de residencia médica en lugares que todavía no los tienen, y en la ampliación y mejora de los programas existentes, pueden ser efectivas (Póvoa, 2006). Para ofrecer condiciones de sustentabilidad, con el objetivo de la continuidad de las acciones de los programas de residencia y la consecuente mejora en la formación de especialistas en el país, la Dirección de Gestión de la Educación en Salud (DEGES) de la Dirección de Gestión Laboral y Educación para la Salud (SGTES) del Ministerio de Salud (MS) empezó en 2009 a implementar una nueva modalidad de financiación para residencias médicas, multiprofesionales y profesionales de la salud, denominada "Pró-Residência" (Ministerio de Salud de Brasil, 2013). El objetivo principal de este programa es el pago de becas para la formación de médicos en especialidades estratégicas, según el estado o región. En 2012, en el estado de Minas Gerais, el Departamento de Salud del estado de Minas Gerais, con el apoyo de Aremg y la Asociación de Hospitales de Minas Gerais, realizó numerosos talleres de capacitación para la creación y registro de programas de residencia médica en hospitales con potencial de capacitación, promovió visitas de médicos con experiencia en supervisión de residencias médicas a instituciones del interior del estado e incluso ofreció incentivos económicos a los hospitales que lograron aprobar programas de residencia medica. Al cabo de 3 años, fueron capaces de aumentar el número de vacantes ofrecidas en un $24 \%$.

Un gran punto a cambiar en Brasil es la inversión en el médico preceptor. No hay reconocimiento de la carrera de preceptor. Considerando que la residencia médica es reconocida como el estándar de oro en la formación de médicos especialistas en el país, este hecho quizás se deba en gran parte a la dedicación personal, disposición y determinación de los preceptores. Muchos de los programas creados hasta ahora fueron inicialmente iniciativa de estos preceptores médicos.

Dada la extrema importancia de la residencia medica en la formación de médicos en Brasil, existe amplia evidencia que le atribuye un doble papel: complementar el proceso de graduación, en vista de las deficiencias ampliamente reconocidas de la educación médica y ofrecer la especialización como posibilidad para mejor inserción en el mercado laboral, constituyendo una forma específica de ingreso 
al mercado. Es posible plantear la hipótesis de que la importancia de la residencia en el proceso de formación de los médicos es mayor y distinta. Parece haberse convertido en el momento que marca con más fuerza el perfil profesional de los médicos jóvenes (Feuerwerker, 1998). En este contexto, tenemos una frase de autor anónimo, pero con gran significado que dice "las escuelas enseñan medicina y la residencia enseña a ser doctor" es decir, no basta con adquirir conocimientos en las escuelas, la estructura de la residencia medica es necesaria para transformar a un recién egresado en un doctor en todo el sentido de la palabra.

En 2018, el CNRM puso en marcha el proyecto de cambio a formación por competencias con el inicio de la publicación de las matrices de competencias a alcanzar al finalizar la formación. El trabajo ocurrió en colaboración con sociedades de especialidades médicas. Una vez finalizada la construcción de las matrices, se inicia la segunda fase con el desarrollo de los instrumentos de evaluación de competencias y su posterior implementación, lo que demandará un gran esfuerzo colectivo ante la necesidad de entender la nueva metodología y los cambios en los parámetros de evaluación por parte de todos los preceptores involucrados en la residencia médica (Tabla 1).

Tabla 1: Historia de la residencia médica en Brasil.

\begin{tabular}{|c|c|}
\hline 1940 & $\begin{array}{l}\text { Creación de la residencia médica en Brasil - inspirada en la } \\
\text { filosofía del modelo norteamericano }\end{array}$ \\
\hline 1967 & Creación de la Asociación de Médicos residentes - ANMR \\
\hline 1977 & $\begin{array}{l}\text { Creación de la Comisión Nacional de Residencia Médica - } \\
\text { CNRM que regula la residencia médica en Brasil }\end{array}$ \\
\hline 1978 & $\begin{array}{l}\text { Creación de la Comisión de Residencia Medica (COREME) que } \\
\text { actúa a su vez como representación del CNRM en la institución }\end{array}$ \\
\hline 1981 & $\begin{array}{l}\text { Publicación de la Ley N } 6932 \text {, el término"residencia médica" } \\
\text { sólo debe utilizarse para los programas acreditados por el CNRM. }\end{array}$ \\
\hline 1987 & Creación los Comités Estatales de Residencia Médica (CEREM) \\
\hline 2002 & $\begin{array}{l}\text { Fundación de la Asociación de Apoyo a la Residencia Médica } \\
\text { de Minas Gerais - AREMG }\end{array}$ \\
\hline 2009 & $\begin{array}{l}\text { Nueva modalidad de financiación para residencias médicas, } \\
\text { multiprofesionales y profesionales de la salud, denominada } \\
\text { "Pró-Residência" }\end{array}$ \\
\hline 2018 & $\begin{array}{l}\text { Cambio a formación por competencias con el inicio de la } \\
\text { publicación de las matrices de competencias }\end{array}$ \\
\hline
\end{tabular}

La residencia médica, por tratarse de la formación en servicio de un profesional imprescindible para la sociedad y de elevado coste económico, acaba por ser una modalidad de posgrado que mantiene un conflicto de intereses entre los distintos actores implicados, intereses muchas veces divergentes. Por un lado, tenemos al prestador de servicios, que puede ser de una institución pública, administrada por diferentes organismos gubernamentales, o una institución filantrópica que brinda servicios al gobierno, o incluso una institución privada con ánimo de lucro. Por otro lado, está el profesional de la salud, el preceptor de la residencia, quien en última instancia será el responsable de la formación en primera línea, el cual actualmente no recibe remuneración por este tipo de actividad y se inserta en su función asistencial y finalmente el médico residente, la parte más vulnerable que busca oportunidades de formación para convertirse en el especialista para ejercer plenamente la profesión. Por encima de todos estos actores está el gobierno federal, el cual, a través del Ministerio de Salud, necesita organizar la formación de recursos humanos que satisfagan las necesidades del país. Tenemos entonces el escenario estándar de las dificultades de conciliar estos intereses a favor del objetivo común, que es formar un buen especialista para brindar los mejores servicios a la sociedad. Inicialmente, tenemos gran dificultad para conciliar la oferta de vacantes que el país necesita según las directrices del sistema único de salud, que es el sistema nacional de atención a la población, y el interés de los egresados de las facultades de medicina. Desde la década de 1990, Brasil ha estado invirtiendo en atención primaria, implementando el Programa de Salud de la Familia y fomentando la creación de programas de residencia médica en Salud de la Familia y la Comunidad, que es el nombre de la especialidad médica en Brasil, pero la especialidad no ha despertado el interés de los médicos recién egresados, entre otros factores podemos mencionar como factor principal el hecho de que no existe una carrera médica consolidada en el área, sin posibilidad de progresión profesional, lo que refleja directamente la dificultad de ampliar la formación de especialistas. Según el CNRM Actualmente, en Brasil se ofrecen 3566 plazas para la residencia en salud familiar y comunitaria en 347 instituciones, todas con becas financiadas por el gobierno y en 2021 solo se ocuparon 1131 vacantes, lo que representó una ociosidad de 68,3\% del total (CNRM, 2021).

En relación a los demás conflictos mencionados, tenemos que la fuerza laboral adicional que representa el médico residente despierta un gran interés de las instituciones ante el alto impacto de la retribución médica en el costo de los servicios de salud. Se suele contratar a un médico por 20 o 24 horas a la semana, asumarse el médico residente con una gran carga de trabajo de actividad que en Brasil es de 60 horas a la semana, por un costo asequible y sin tarifas adicionales de la legislación laboral o incluso los medios de becas del Gobierno, resultando en ahorros significativos para las instituciones. Mientras tanto, los preceptores que guiarán la actividad diaria del residente generalmente no son remunerados directamente por la función, pero vislumbran en la actividad un intercambio en el que el médico residente realiza gran parte de su labor, llegando en muchas instituciones, a realizar la labor de varios 
médicos en una clara distorsión del papel de la residencia medica y generando conflictos. En cuanto al médico residente, tenemos una doble figura de profesional con muchos años dedicados a la formación médica, muchas veces con un alto costo para la familia $y$, por otro lado, en el rol de estudiante recibiendo una beca de un monto limitado e insuficiente para el costeo de una vida cómoda a la que aspira como profesional. A menudo se ve impulsado a buscar actividades adicionales para complementar sus ingresos a expensas de la formación en la especialidad.

Tenemos entonces el gran desafío de conciliar los distintos intereses en un equilibrio que garantice la formación de un especialista con las competencias requeridas para el ejercicio profesional con el fin de cumplir con la actividad principal, que es la prestación de servicios de calidad y seguros a la población. En aproximadamente 40 años, se construyó en Brasil un gran sistema de formación de médicos especialistas, que actualmente involucra a 1218 instituciones acreditadas que capacitaron a 15171 especialistas en 2021. Es un sistema en constante evolución, en un equilibrio muy sensible, enfrentando los desafíos de ser guiados por conceptos técnicos que independen de las decisiones políticas que influyen en todo el sistema.

\section{Referencias}

Asociación de Apoyo a la Residencia Médica de Minas Gerais AREMG. (2021) Proceso de selección unificado para la residencia médica. Accedido en: http://www.aremg.org.br/processos-atuais el 20 de noviembre de 2021.

Asociación Nacional de Médicos Residentes - ANMR. (2013) Antecedentes [Internet]. [Accedido en: 26 de marzo de 2013]. Revista Ciencias em Salud v3, n1, enero - marzo de 2013 Accedido en: http://www.anmr.org/index.php?formulario = histórico \& metodo $=0 \& \mathrm{id}=1$ el 02 de octubre de 2021.

Comisión Nacional de Residencia Médica - CNRM - (2021). Las nuevas vacantes benefician a los estados en las regiones con mayor necesidad. Accedido en: http://portal.mec.gov.br/component/tags/tag/comissao-nacional-de-residencia-medica el 10 de septiembre de 2021

Boletín Oficial de La Unión, 1988. Constitución de la República Federativa de Brasil. Accedido en: https://www2.camara.leg.br/legin/ fed/consti/1988/constituicao-1988-5-outubro-1988-322142-publicacaooriginal-1-pl.html el 09 de septiembre de 2021.

Feuerwerker L. (1998) Cambios en la educación médica y la residencia médica en Brasil. Interfaz: comunicación, salud, educación 2, 51-71.
Instituto Brasileño de Geografía y Estadística - IBGE. (2021). Proyección de la Población de Brasil y Unidades Federación. Accedido en: https://www.ibge.gov.br/apps/populacao/projecao/index.html el 10 de septiembre de 2021 .

Martins LAN. (2005) Residencia médica estrés y crecimiento. Revista Brasileña de Psiquiatría - ABP 28, 307-308.

Medidores de países. (2021). Población de palabras clave y países, Accedido en: https://countrymeters.info/pt/South_America el 09 de septiembre de 2021.

Ministerio de Educación de Brasil (2021). Registro Nacional de Cursos e Instituciones de Educación Superior. Registro e-MEC. Accedido en: https://emec.mec.gov.br/ el 14 de septiembre de 2021.

Ministerio de Educación de Brasil. (2011) Leyes, decretos, etc. Decreto $N^{\circ} 7.562$, de 15 de septiembre de 2011. Establece la Comisión Nacional de Residencia Médica y el ejercicio de las funciones de regulación, supervisión y evaluación de las instituciones que ofrecen programas de residencia médica y residencia médica. Brasilia: Boletín Oficial de la Unión; 2011. Accedido en: http://www.planalto.gov.br/ccivil_03/_ ato2011-2014/2011/decreto/d7562.htm el 4 de octubre de 2021.

Ministerio de Educación de Brasil. (1977) Leyes, decretos, etc. Decreto núm. 80.281 del 5 de septiembre de 1977. Regula la Residencia Médica, crea la Comisión Nacional de Residencia Médica y establece otras disposiciones. Brasilia: Boletín Oficial de la Unión; 1977. Accedido en: http://portal.mec.gov.br/sesu/arquivos/pdf/ d80281.pdf el 4 de octubre de 2021.

Ministerio de Educación de Brasil. (1981) Leyes, decretos, etc. Ley no 6. 932, de 7 de julio de 1981. Establece las actividades de los médicos residentes y otras medidas. Brasilia: Boletín Oficial de la Unión; 1981. Accedido en: http://www.planalto.gov.br/ccivil_03/ leis/l6932.htm el 5 de octubre de 2021.

Ministerio de Educación de Brasil. (2006) Departamento de Educación Superior. Resolución CNRM 01, de 3 de enero de 2006. Establece la estructura, organización y funcionamiento de los Comités Estatales de Residencia Médica. Brasilia: Boletín Oficial de la Unión; 2006. Accedido en: http://portal.mec.gov.br/index. php?option=com_docman\&view=download\&alias=508-resolucao-cnrm-01-03012006\&category_slug=documentos-pdf\&ltemid=30192 el 14 de septiembre de 2021.

Ministerio de Educación de Brasil. (2010) Resolución CNRM No. 4, de 12 de julio de 2010. Prohíbe la guardia a los Médicos Residentes en el ámbito de la Residencia Médica. Brasilia: Boletín Oficial de la Unión; 2010. Accedido en: http://portal.mec.gov.br/index. 
php?option=com_docman\&view=download \&alias=100881-resolucao-cnrm-n-4-de-12-de-julho-de-2010\&category_slug=november -2018-pdf \& Itemid = 30192 el 10 de octubre de 2021.

Ministerio de Educación de Brasil. (2011). Ley $N^{\circ} 12.514$, de 28 de octubre de 2011. Conversión de la Medida Provisional N ${ }^{\circ} 536$, de 2011. Da nueva redacción al art. 4 de la Ley $N^{\circ} 6.932$, de 7 de julio de 1981, que regula la actividad de los médicos residentes. Brasilia: Boletín Oficial de la Unión; 2011. Accedido en: http://www. planalto.gov.br/ccivil_03/leis/16932.htm el 3 de octubre de 2021.

Ministerio de Educación de Brasil. (2012) Secretaría de Educación Superior. Noticia publica no 5, de 20 de abril de 2012 - proceso de selección para la conformación de un padrón de especialistas para la composición del banco público de evaluadores de la comisión nacional de residencia médica. Brasilia: Boletín Oficial de la Unión; 2012. Accedido en: http://portal.mec.gov.br/programa-mais-educacao/247-programas-e-acoes-1921564125/residencia-medica-2137156164/12500-Ley especifica el 15 de septiembre de 2021.
Ministerio de Salud de Brasil. Portal de Salud. Pro-Residencia. Accedido en: http://portal.saude.gov.br/portal/saude/profissional/ area.cfmid_area=1748 el 10 de octubre de 2021.

Póvoa L. y Andrade MV. (2006) Distribución geografía de los médicos en Brasil: un análisis basado en un modelo de elección de ubicación. Salud Pública Cad 22, 1555-64.

SIB /ANS/MS. (2021) Sistema de Información de Beneficiarios. Beneficiarios de planes de salud privados, para cobertura asistencial. Accedido en: https://www.ans.gov.br/perfil-do-setor/dados-gerais

Sousa EG. (1988) Consideraciones sobre la residencia médica en Brasil. Rev. Bras Colo-Proct. 8, 150-2.

Universidad de São Paulo - USP (2018) - Demografía médica en Brasil en 2018 - Accedido en: https://eephcfmusp.org.br/portal/ online/residencia-medica/ el 09 de septiembre de 2021. 